\title{
Influences of Stimulation Conditions on Recruitment of Myelinated Nerve Fibers: A Model Study
}

\author{
PETER H. VELTINK, JAN A. VAN ALSTÉ, MEMBER, IEEE, \\ AND HERMAN B. K. BOOM
}

\begin{abstract}
The dependency of excitation of myelinated nerve fibers on nerve-fiber diameter, and the geometrical spread of recruited nerve fibers in the fascicle during artificial nerve stimulation was investigated by varying parameters of a simulation model presented in a previous paper. The model predicts that fascicle conductivities and the connective tissue sheath surrounding the fascicle, the extraneural tissue, together with the radius of the fascicle and the thickness of the connective tissue sheath, influence both recruitment order and geometrical spread of recruited motoneuron fibers in the fascicle.

The model predicts recruitment probability to increase for increasing nerve-fiber diameter in the entire, or almost entire range of stimulation pulse-amplitudes in all simulated cases. In a probabilistic sense, this implies an inverse order of recruitment compared to the physiological order. It does not always necessarily imply inverse recruitment in realizations of the stochastic process. Simulation results are in correspondence with inverse recruitment reported for extraneural stimulation and a mixed order of recruitment found for intrafascicular stimulation: the model predicts this more pronounced inverse recruitment for extraneural electrodes for all parameter combinations of the volume conductor considered.
\end{abstract}

\section{INTRODUCTION}

$\mathrm{R}^{\mathrm{s}}$ ESTORING the function of paralyzed extremities of spinal cord-injured patients by means of artificial stimulation of the peripheral neuromuscular system is of growing interest. Peripheral nerve stimulation for controlling contraction of skeletal muscles is felt to have potential for implantable stimulation systems. The conventional approach of nerve stimulation is one-channel extraneural stimulation, mostly using a loose-fitting isolation cuff with two ring electrodes for bipolar stimulation. This method recruits thicker nerve fibers before thin, an inverse order of recruitment as compared to the physiological situation [1]: Blair and Erlanger [2] found inverse recruitment for extraneural stimulation of frog sciatic nerve by single-nerve fiber recordings, while stimulating with bipolar extraneural electrodes. Petrofsky [3], stimulating the ventral roots of a cat using extraneural bipolar ring electrodes, and Fang and Mortimer [4], stimulating a branch of the sciatic nerve in a cat via a bipolar cuff configuration, also showed inverse recruitment by measuring muscle twitch widths. Solomonow [5], stimulating the sciatic nerve of a cat with a bipolar cuff config-

\footnotetext{
Manuscript received November 16, 1987; revised June 20, 1988

The authors are with the Biological Engineering Division, Department of Electrical Engineering, University of Twente, Enschede, The Netherlands.
}

IEEE Log Number 8823294 uration, showed inverse recruitment by demonstrating a higher threshold for the activation of the predominantly slow soleus muscle, as compared to faster gastrocnemius muscle, while soleus threshold was lower than gastrocnemius threshold when using high-frequency blocking for restoring physiological order.

Experiments of Veltink et al. [6], [7] on intrafascicular stimulation of a rat's common peroneal nerve showed a mixed order of recruitment. Small wire electrodes, isolated up to the very tip and placed inside the fascicle were used for stimulation. This mixed order of recruitment was indicated by differing distances between force levels in the recruitment curve (relation between stimulation pulseamplitude and isometrical muscle twitch force), measured from tibialis anterior and extensor digitorum longus muscles. These distances correspond to discrete motor unit contributions.

Much research in the field of artificial nerve stimulation is directed towards obtaining a control of muscle contraction that is similar to physiological control. One approach is to restore physiological recruitment order by blocking motoneurons recruited first (Petrofsky and Phillips [8], Solomonow [9], and Fang and Mortimer [4]). These authors found an improved fatigue resistance during isometrical muscle contraction when the physiological recruitment order was restored.

Apart from restoring physiological recruitment order, investigations into the use of multielectrode configurations for improving the performance of nerve-stimulation methods have been described [10], [11], [12], [13]. Holle [10] and Petrofsky [11] investigated the alternating activation of disjunct groups of motoneurons and the associated motor units using a multielectrode configuration, and found that it could postpone muscle fatigue. Furthermore, McNeal and Bowman [12] and Talonen et al. [13] found that selective activation of different muscles with different electrodes of an extraneural multielectrode configuration is possible when motoneurons are geometrically ordered in the nerve according to the muscles they innervate.

With multielectrode nerve stimulation it is optimal that with every electrode a unique group of motoneurons is excited, which does not overlap with motoneuron groups excited by other electrodes. In order to decrease functional overlap of the motoneuron groups recruited by different electrodes, a minimal geometrical spread of the motoneurons recruited by the same electrode is desirable. 
Veltink et al. [14] proposed a model of nerve-fiber excitation by volume conduction of electrical stimuli in a cylindrical, one-fascicular nerve geometry. It included statistical distributions for nerve-fiber position, nerve-fiber diameter, and node position. The model showed that under the implied assumptions nonstrict inverse recruitment of motor units is possible, due to geometrical conditions. However, this model does not make clear how general and, therefore, how likely such a situation is or to what extent the inverse recruitment is due to the specific choice of parameters. Therefore, influences of the volume conduction characteristics on diameter dependent recruitment of myelinated nerve fibers and on geometrical spread of motoneurons recruited by a single electrode were investigated by simulations with the model. Parameters as fascicle radius, thickness of the connective tissue sheath surrounding a fascicle, and the conductivities of the media inside and outside the nerve were systematically varied. This sensitivity analysis is reported in the present paper. In general, extraneural stimulation is more probable to yield strictly inverse recruitment than intrafascicular stimulation.

\section{Methods \\ Probabilistic Nerve-Stimulation Model}

The starting point of work presented in this paper will be the nerve-stimulation model proposed by Veltink $e t$ al. [14], assuming a cylindrical symmetric nerve geometry ( radius $r_{o}$ ) with one fascicle, represented by the medium $i$ within the inner cylinder with radius $r_{i}$ (Fig. 1) [14]. This medium contained the myelinated motoneurons to be stimulated and was assumed to have an anisotropic conductivity with parallel and transverse conductivities $\sigma_{\mathrm{iz}}$ and $\sigma_{\text {ir }}$. The connective tissue sheath surrounding the fascicle (medium $o$ in between both cylinders) and the extraneural medium outside the outer cylinder were assumed to have an isotropic homogeneous conductivity $\sigma_{o}$ and $\sigma_{e}$, respectively. Two electrode configurations were considered: an intrafascicular point electrode at position $r=0, z=0$ and an extraneural ring electrode at $r=r_{o}, z=0$ [14]. Stimulation was monopolar cathodic with a rectangular current-pulse of $60 \mu \mathrm{s}$ width. In this study only amplitude $A$ was varied, although varying pulse width or pulse form may influence recruitment properties [15].

The electrical potential in the nerve was calculated by solving Laplace's equation in cylindrical coordinates, as described by Veltink et al. [14]. Modeling of nerve-fiber excitation was based on McNeal [16], extended by modeling three nodes of Ranvier by the Frankenhaeuser-Huxley equations [17]. Like McNeal, a linear relation between nerve-fiber diameter $D$ and internodal length $L$ was assumed. Furthermore, in our model, the node of Ranvier nearest to the stimulation electrode was not necessarily positioned in the current-injection plane perpendicular on the nerve fibers. Consequently, the thickest nerve fiber did not necessarily have the lowest excitation threshold of all nerve fibers at the same distance from the electrode.

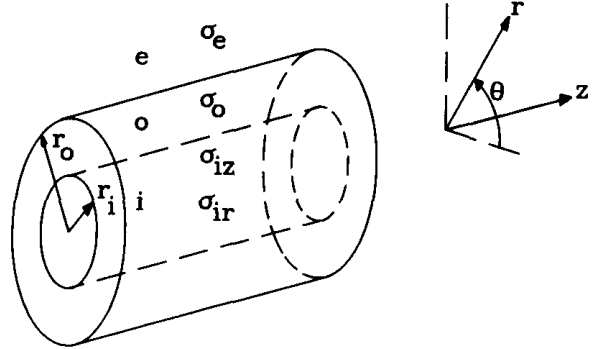

Fig. 1. Two-cylinder model of a nerve [14]. The inner cylinder represents the boundary of the fascicle $i$ with radius $r_{i}$ and conductivity $\sigma_{i z}$ in $z$ direction and $\sigma_{\mathrm{ir}}$ in $r$ direction. A connective tissue sheath is represented by the medium in between both cylinders. The electrical conductivity of this medium is $\sigma_{o}$ and the thickness $r_{o}-r_{i}$. Outside the outer cylinder, an extraneural medium with conductivity $\sigma_{e}$ is modeled.

Veltink et al. [14] described a number of parameters of the nerve-fiber excitation model not known a priori by random variables. These included $D$, representing the fiber diameter; $\boldsymbol{r}$, its radial position; $\boldsymbol{L}_{\mathbf{0}}$, the distance from current-injection plane to its nearest node of Ranvier; and $\boldsymbol{A}=A\left(D, r, L_{0}\right)$ being the stimulation pulse amplitude above which the nerve fiber is excited. By assuming probability distribution functions for $\boldsymbol{D}, \boldsymbol{r}$, and $\boldsymbol{L}_{\mathbf{0}}$, expressions for the probability of a nerve fiber being excited at a given stimulation pulse amplitude $A$ have been described by Veltink et al. [14]. The dependency of nerve-fiber excitation on the fiber's radial position $r$ and diameter $D$ and on stimulus pulse amplitude $A$ will especially be discussed. To this end, the probability density of excitation $g_{A \mid D}(r)$ for a given diameter $D$ at stimulation pulse amplitude $A$ was used, as was shown in our previous paper [14] and illustrated in Fig. 2(a). The diagonal line in Fig. 2(a) represents excitation of all nerve fibers and corresponds to the probability density function of $r$. The distribution of the nerve fibers over the cross section of the nerve was supposed to be uniform. Also the node position $L_{0}$ was supposed to be uniformly distributed between $-L / 2$ and $L / 2$ ( $L$ is the internodal length). Furthermore, the probability distribution $F_{A \mid D}(A \mid D)$ has been defined [14] [Fig. 2(b)] as the probability of excitation of any fiber of diameter $D$ as a function of $A$. It is related to the density function $g_{\boldsymbol{A} \mid \boldsymbol{D}}(r)$ by

$$
\left.F_{A}\right|_{D}(A \mid D)=\int_{0}^{r_{i}} g_{A \mid D}(r) \cdot d r
$$

The distribution function $F_{A \mid D}(A \mid D)$ can be interpreted as a recruitment curve for given diameter nerve fibers.

\section{Variation of Model Parameters}

The influences of variations of the parameters $\sigma_{e}, \sigma_{o}$, $\sigma_{\mathrm{iz}}, \sigma_{\mathrm{ir}}, r_{i}$ and $r_{o}-r_{i}$ on the recruitment properties of nerve fibers of different diameters $(5,10,15$, and $20 \mu \mathrm{m})$ was investigated. Two values of each model parameter were considered (see Table I) of which all combinations were investigated in this study. Set $A$ consists of the lowest parameter values, set $B$ of the highest. 


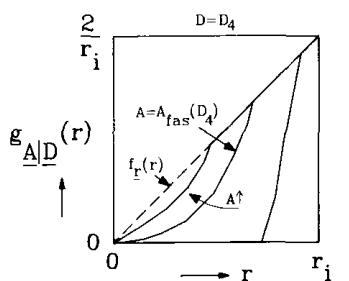

(a)

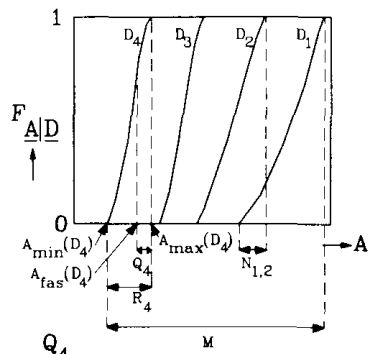

(b)
Fig. 2. Illustration of the functions and variables defined for evaluation of the simulation results. Probability distribution $F_{A \mid D}$ (b) is obtained by integrating the probability density of excitation $g_{A \mid D}(r)$ (a) over the fascicle radius for several values of pulse amplitude $A$. The diagonal line in (a) represents excitation of all nerve fibers and corresponds to the probability density function $f_{r}(r) . A_{\min }, A_{\text {fas }}$, and $A_{\max }$ are defined in the text and illustrated in this figure for an extraneural electrode.

TABLE I

The Two Sets of Parameter Values Considered

\begin{tabular}{cc|c|c|}
\hline \hline \multicolumn{2}{|c|}{ model parameter } & $\begin{array}{c}\text { parameter } \\
\text { set A }\end{array}$ & $\begin{array}{c}\text { parameter } \\
\text { set B }\end{array}$ \\
\hline$\sigma_{\mathrm{e}}$ & {$\left[(\Omega \mathrm{m})^{-1}\right]$} & 0.01 & 0.04 \\
\hline$\sigma_{0}$ & {$\left[(\Omega \mathrm{m})^{-1}\right]$} & 0.01 & 0.10 \\
\hline$\sigma_{\mathrm{iz}}$ & {$\left[(\Omega \mathrm{m})^{-1}\right]$} & 0.25 & 0.50 \\
$\sigma_{\mathrm{ir}}$ & {$\left[(\Omega \mathrm{m})^{-1}\right]$} & 0.08 & 0.08 \\
\hline $\mathrm{r}_{\mathrm{i}}$ & {$[\mathrm{mm}]$} & 0.25 & 0.50 \\
\hline $\mathrm{r}_{0}{ }^{-\mathrm{r}_{\mathrm{i}}}$ & {$[\mathrm{mm}]$} & 0.05 & 0.50 \\
\hline
\end{tabular}

The parameter value $\sigma_{e}^{A}$ in set $A$ represents a nearly isolating medium around the nerve, $\sigma_{e}^{B}$ models a nerve surrounded by fat tissue, as can be assumed to be the case when a nerve is in direct contact with its surrounding medium [10], [18]. The conductivity of fat tissue was obtained from Geddes and Baker [19] $\left(0.04(\Omega \cdot \mathrm{m})^{-1}\right)$.

No conductivity value for the connective tissue sheath $\sigma_{o}$ was found in literature. It is difficult to establish an unambiguous value because the composition of the connective tissue sheath is not very homogeneous [20]. The structure is denser around both the fascicle and at the surface of the nerve as compared to the bulk of the connective tissue sheath. Furthermore, several structures, like blood vessels, can lie in the connective tissue sheath. We used two values of $\sigma_{o}$ differing by a factor 10 , the one higher and the other lower than the conductivity of fat tissue.

The anisotropic intrafascicular conductivity in set $B$ was obtained from Geddes and Baker [19]. To evaluate the influence of anisotropy inside the fascicle, $\sigma_{\mathrm{iz}}^{A}$ was taken half of $\sigma_{i z}^{B}$.

According to Sunderland [20], the radius of a fascicle can vary between 0.02 and $1.7 \mathrm{~mm}$. Radii $r_{i}^{A}$ and $r_{i}^{B}$ were chosen as shown in Table I because the radius of most fascicles will be in the middle of this range. Radius $r_{i}^{A}$ corresponding to the radius of the fascicle inside the rat's common peroneal nerve which was used in experiments described by Veltink et al. [6], [7].

The distance from the surface of the nerve to the fascicle can vary considerably between nerves. Only a thin connective tissue sheath surrounds the fascicle of the rat's common peroneal nerve used by Veltink et al. [6], [7]. The value of $r_{o}^{A}-r_{i}^{A}$ reflects this situation, while the $B$ value corresponds to a fascicle in the middle of a larger nerve.

\section{Evaluation of Simulation Results}

Three characteristic stimulation pulse amplitudes were determined for all combinations of the parameter values for every nerve-fiber diameter considered and for both electrode configurations (Fig. 2):

- $A_{\text {min }}(D)$ being the stimulation pulse amplitude at which recruitment of nerve fibers with diameter $D$ starts.

- $A_{\text {fas }}(D)$ being the minimum stimulation pulse amplitude at which nerve fibers of diameter $D$ may be recruited throughout the fascicle.

- $A_{\max }(D)$ being the minimum stimulation pulse amplitude at which all nerve fibers with diameter $D$ are recruited.

$A_{\min }(D)$ was determined by simulating a nerve fiber with diameter $D$ lying as close as possible to the stimulation electrode ( $r=0$ for the intrafascicular electrode and $r=r_{i}$ for the extraneural electrode), and with the most favorable position of the nearest node of Ranvier ( $L_{0}$ $=0$ ). For the intrafascicular electrode, $A_{\min }(D)$ is always zero because the extracellular potential at the node at $z=$ 0 will be higher than every threshold when $r$ reaches 0 (the electrode is indefinitely small).

$A_{\text {fas }}(D)$ was established by simulating a nerve fiber with diameter $D$ lying farthest away from the electrode $\left(r=r_{i}\right.$ for the intrafascicular and $r=0$ for the extraneural electrode), and with the most favorable position of the nearest node: $L_{0}=0$.

$A_{\max }(D)$ was determined by simulating a nerve fiber with diameter $D$ at the same radial position as used for computing $A_{\text {fas }}(D)$. However, the value of $L_{0}$ now was taken most unfavorable: $L_{0}=L(D) / 2$.

Furthermore, the following variables were defined for the evaluation of the simulation results (Fig. 2):

- Geometrical spread of the recruited nerve fibers, as described by the density function $g_{A \mid D}(r)$, was represented by $S_{i}$ :

$$
\begin{aligned}
S_{i}=\frac{A_{\mathrm{max}}\left(D_{i}\right)-A_{\mathrm{fas}}\left(D_{i}\right)}{A_{\max }\left(D_{i}\right)-A_{\min }\left(D_{i}\right)} & \\
& \quad i=1,2,3,4, \quad D_{i}=5,10,15,20 \mu \mathrm{m} .
\end{aligned}
$$

$S_{i}=1$ holds when recruitment probability is greater than 0 in the whole fascicle already at threshold-stimulation $A_{\min }\left(D_{i}\right)$ (maximal spread). $S_{i}=0$ indicates a recruitment 
probability greater than 0 in the whole fascicle only at full recruitment $A \geq A_{\max }\left(D_{i}\right)$ (minimal spread).

- Simultaneousness of the recruitment curves for different diameter nerve fibers described by $F_{A \mid D}(A \mid D)$ is indicated by variable $T_{i, j}$ :

$$
T_{i, j}=\frac{N_{i, j}}{M} \quad i, j=1,2,3,4, i \neq j .
$$

- $M$ is the amplitude range in which recruitment probability is unequal to 0 or 1 for at least one of the four nerve-fiber diameters considered [Fig. 2(b)].

- $N_{i, j}=A_{\max }\left(D_{j}\right)-^{-} A_{\min }\left(D_{i}\right)$ is the amplitude range in which recruitment probability for both nerve-fiber diameters $D_{i}$ and $D_{j}\left(D_{k}=5,10,15,20 \mu \mathrm{m}\right)$ is unequal to 0 or 1 .

$T_{i, j}$ gives the amplitude range in which either nerve fibers of diameters $D_{i}$ or $D_{j}$ could additionally be recruited when the stimulation pulse amplitude is increased. It is normalized with respect to the total amplitude range in which any nerve fiber of the four diameters considered can be recruited additionally for increasing stimulation pulse amplitude. $T_{i, j}$ can be determined from $A_{\min }$ and $A_{\max }$ for the four nerve-fiber diameters considered.

For positive values of $T_{i, j}$, there is an amplitude range in which nerve fibers of either diameters $D_{i}$ and $D_{j}$ could additionally be recruited when stimulus pulse amplitude $A$ increases. This is not the case for negative $T_{i, j}$ in which case the value of $T_{i, j}$ indicates the distance between the stimulation pulse amplitude ranges in which recruitment is possible for either of the nerve-fiber diameters $D_{i}$ or $D_{j}$. Because $A_{\min }(D)=0$ holds for all $D$ in the case of the intrafascicular electrode, the variable $T_{i, j}$ will always be greater than 0 for the intrafascicular electrode.

\section{RESUlTS}

Simulated values for the functions $g_{A \mid D}(r)$ and $F_{A \mid D}(A \mid D)$ are shown in Figs. 3 and 4 for two cases, with parameter values and electrode configurations as specified in Table II. The values of $A_{\min }, A_{\text {fas }}, A_{\max }, S_{i}$, and $T_{i, i+1}$ are given in Table III.

The first case [Figs. 3(a) and 4(a)] is an intrafascicular electrode and a nerve geometry that corresponds best to the geometry of the small common peroneal nerve of a rat used in the experiments of Veltink et al. [6], [7]. In these experiments, the fascicle radius was about $0.25 \mathrm{~mm}$. A thin connective tissue sheath and a low extraneural conductivity was present. Although recruitment probability increases with nerve-fiber diameter, the distribution $F_{A \mid D}(A \mid D)$ in Fig. 4(a) indicates a nonstrict inverse recruitment especially for larger nerve-fiber diameters $(20$, 15 , and $10 \mu \mathrm{m}$ ) because at increasing stimulation pulse amplitude additional recruitment may occur for all four nerve-fiber diameters when $F_{A \mid D}(A \mid D)$ is not constant for all four diameters considered. This nonstrict inverse recruitment corresponds with the mixed order of recruitment for intrafascicular electrodes, which Veltink et al. [6], [7] found in their experiments.

The second case, illustrated in Figs. 3(b) and 4(b),

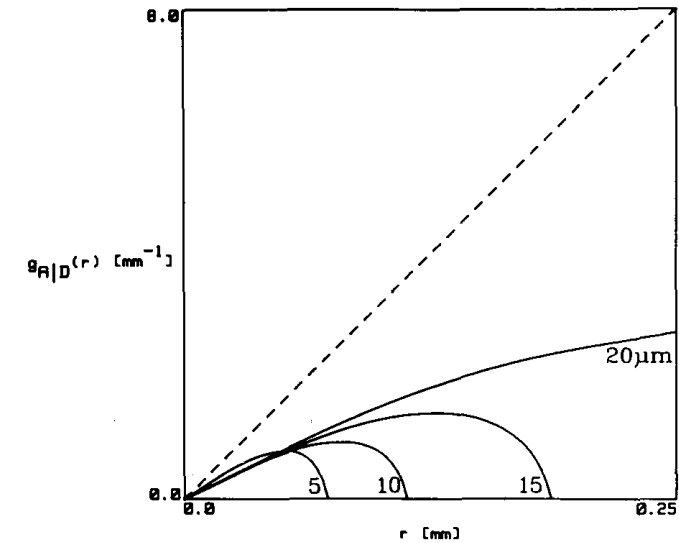

(a)

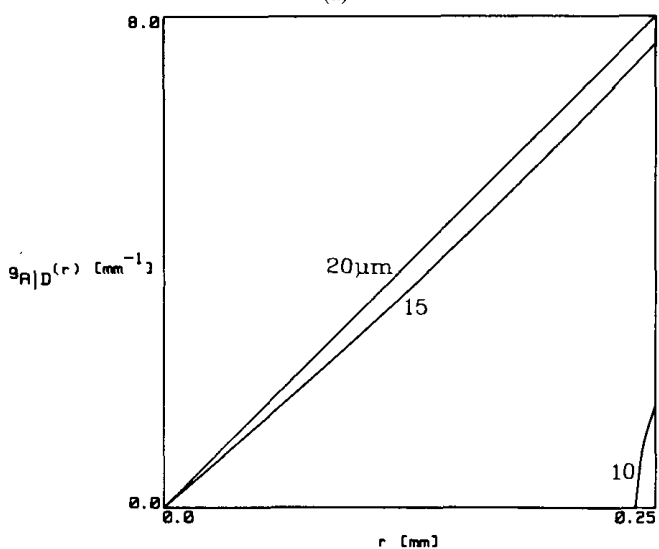

(b)

Fig. 3. Functions $g_{A}(r)$ for intrafascicular and extraneural stimulation. The parameters are specified in Table II. Functions $g_{A \mid D}(r)$ are shown for nerve-fiber diameters $5,10,15$, and $20 \mu \mathrm{m}$ at a single stimulation pulse amplitude $A$. (a) Intrafascicular stimulation, $A=9 \mu \mathrm{A}$. (b) Extraneural stimulation, $A=30 \mu \mathrm{A}$.

shows $g_{A \mid D}(A \mid D)$ and $F_{A \mid D}(A \mid D)$ for an extraneural ring electrode and a thicker connective tissue sheath. The other parameters are the same as in Figs. 3(a) and 4(a). A much more pronounced, although not necessarily absolute, inverse recruitment as compared with the first example is predicted by the model, which means relatively low values of $T_{i, i+1}$ (Table III).

The ranges $A_{\max }\left(D_{i}\right), S_{i}$, and $T_{i, i+1}$, together with their mean values, are shown in Fig. 5 for all parameter combinations. Mean values of $A_{\max }\left(D_{i}\right)$ and $S_{i}$ are higher for the extraneural electrode than for the intrafascicular electrode. As described, $T_{i, i+1}$ is always positive for intrafascicular electrodes. For the extraneural electrode, however, negative values of $T_{i, i+1}$ were also found, especially for small nerve-fiber diameters, indicating strict inverse recruitment for the two nerve-fiber diameters considered.

Not shown in Fig. 5 are the following results:

1) All cases for extraneural stimulation with large distance between electrode and fascicle $\left(r_{o}-r_{i}=0.5 \mathrm{~mm}\right)$ had negative values of $T_{1,2}(5 / 10 \mu \mathrm{m})$. 


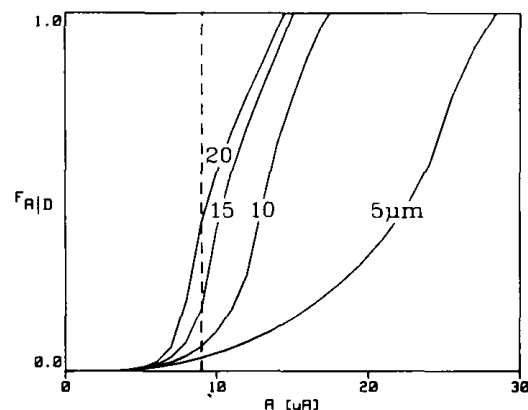

(a)

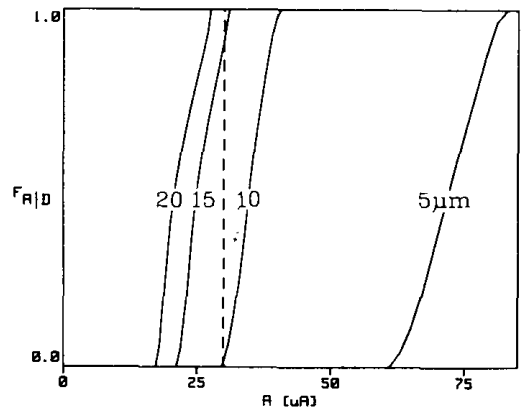

(b)

Fig. 4. Examples of the distribution functions $F_{A \mid D}(A \mid D)$ for intrafascicular (a) and extraneural (b) stimulation. The model parameter combinations are specified in Table II. The dashed vertical lines mark the stimulation pulse amplitudes used in Fig. 3 .

TABLE II

Specification of the Parameter Combinations and the Electrode Configurations in the Examples iv Figs. 3 and 4. The Figures Suffixes A and B Indicate the Parameter Combination Used.

\begin{tabular}{|c|c|c|}
\hline & \multicolumn{2}{|c|}{ example } \\
\hline & A & B \\
\hline $\begin{array}{l}\text { electrode } \\
\text { configuration }\end{array}$ & $\begin{array}{l}\text { intra- } \\
\text { fascicular }\end{array}$ & $\begin{array}{c}\text { extra- } \\
\text { neural }\end{array}$ \\
\hline$\sigma_{\mathrm{e}} \quad\left[(\Omega \mathrm{m})^{-1}\right]$ & 0.01 & 0.01 \\
\hline$\sigma_{0} \quad\left[(\Omega m)^{-1}\right]$ & 0.10 & 0.10 \\
\hline$\sigma_{\mathrm{iz}} \quad\left[(\Omega \mathrm{m})^{-1}\right\}$ & 0.50 & 0.50 \\
\hline$\sigma_{\text {ir }} \quad\left[(\Omega \mathrm{m})^{-1}\right]$ & 0.08 & 0.08 \\
\hline $\mathbf{r}_{\mathbf{i}} \quad[\mathrm{mm}]$ & 0.25 & 0.25 \\
\hline${ }^{\mathrm{r}_{0}}{ }^{-\mathrm{r}_{\mathrm{i}}} \quad[\mathrm{mm}]$ & 0.05 & 0.50 \\
\hline
\end{tabular}

2) $T_{i, i+1}$ appeared to be always smaller for extraneural electrodes than for intrafascicular electrodes for the same volume conductor parameters, indicating a more pronounced inverse recruitment for extraneural electrodes than for intrafascicular electrodes. Furthermore, the differences of $T_{i, i+1}$ between intrafascicular and extraneural electrodes are bigger for a larger $r_{o}-r_{i}$.
TABLE III

Values of the Evalution Variables for the Four Nerve Fiber Diameters Considered in the Case of the Two Example Parameter COMBinations SPECIFIEd IN TABLE II

\begin{tabular}{c|c||c|c|c|c|c|}
\hline \hline example & $\begin{array}{c}\mathrm{D} \\
{[\mu \mathrm{m}]}\end{array}$ & $\begin{array}{c}\mathrm{A}_{\min } \\
{[\mu \mathrm{A}]}\end{array}$ & $\begin{array}{c}\mathrm{A}_{\mathrm{fas}} \\
{[\mu \mathrm{A}]}\end{array}$ & $\begin{array}{c}\mathrm{A}_{\max } \\
{[\mu \mathrm{A}]}\end{array}$ & $\mathrm{S}_{\mathrm{i}}$ & $\mathrm{T}_{\mathrm{i}, \mathrm{i}+1}$ \\
\hline \hline $\mathrm{a}$ & 5 & 0 & 25.4 & 28.4 & 0.11 & 0.61 \\
& 10 & 0 & 13.0 & 17.4 & 0.25 & 0.53 \\
& 15 & 0 & 9.6 & 15.1 & 0.36 & 0.51 \\
& 20 & 0 & 8.2 & 14.4 & 0.43 & \\
\hline $\mathrm{b}$ & 5 & 60.8 & 80.1 & 83.2 & 0.14 & \multirow{6}{*}{} \\
& 10 & 29.5 & 35.2 & 40.7 & 0.50 & 0.30 \\
& 15 & 21.1 & 24.0 & 31.1 & 0.71 & 0.02 \\
& 20 & 17.2 & 19.1 & 27.5 & 0.82 & $\theta .10$ \\
\hline
\end{tabular}

INTRAFASCICULFR POINT ELECTRODE

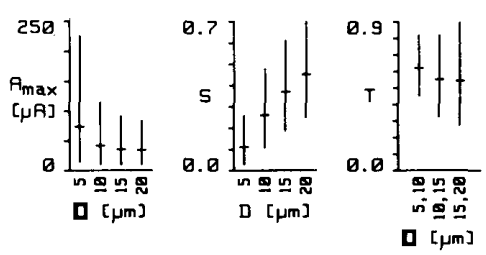

(a)

EXTRFNEURAL RING ELECTRODE

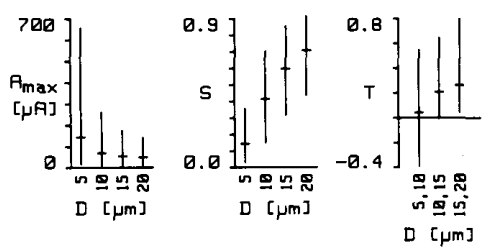

(b)

Fig. 5. Range and mean value of $A_{\max }\left(D_{i}\right), S_{i}$, and $T_{i, i+1}$ for all $32 \mathrm{com}$ binations of the parameter values presented in Table I and both electrode configurations [(a) intrafascicular electrode, (b) extraneural electrode] The vertical bars connect minimum and maximum values. The horizontal marks give the mean values.

Ranges of differential diameter dependencies of $A_{\max }\left(D_{i}\right), S_{i}$, and $T_{i, i+1}$ taken over all parameter combinations are given in Fig. 6 . By this way of presentation it is shown that increasing nerve-fiber diameter always appeared to result in increased geometrical spread $S_{i}$ for any parameter combination and both electrode configurations $(\Delta S>0) . A_{\max }\left(D_{i}\right)$ decreased with increasing fiber diameter, except for large diameters $(15$ and $20 \mu \mathrm{m})$ in a number of cases. This exception indicates larger recruitment probabilities for $15 \mu \mathrm{m}$ than for $20 \mu \mathrm{m}$ diameter. However, this only occured for almost full recruitment ( $F_{A \mid D}$ nearly 1): In all cases, $A_{\text {fas }}$ appeared to be lower for larger nerve-fiber diameter (not shown in the figure).

Fig. 7 shows the influence of each of the model parameters seperately on $A_{\max }\left(D_{i}\right), S_{i}$, and $T_{i, i+1}$ when the parameters are varied from the values in set $A$ to the higher values in set $B$ (Table I). It shows that the influence of $\sigma_{e}$ 
INTRAFASCICULAR POINT ELECTRODE

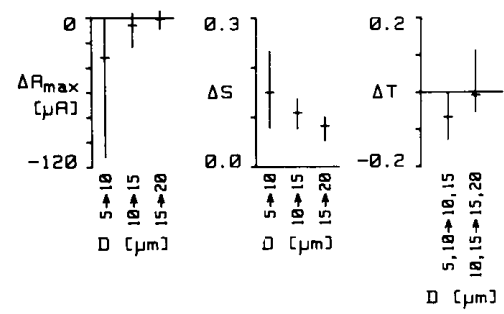

(a)

EXTRANEURAL RING ELECTRODE

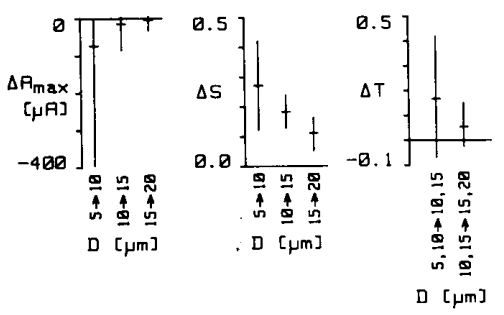

(b)

Fig. 6. The influence of nerve-fiber diameter on $A_{\max }\left(D_{i}\right), S_{i}$, and $T_{i, i+1}$. The changes in value of these variables are given for changing the diameter to a $5 \mu \mathrm{m}$ higher value. The vertical bars connect minimum and maximum value change. The horizontal marks give the mean change for all combinations of the model parameters. (a) Intrafascicular electrode, (b) extraneural electrode.

is relatively high: $A_{\max }\left(D_{i}\right), S_{i}$, and $T_{i, i+1}$ all increase when $\sigma_{e}$ increases from $0.01(\Omega \cdot \mathrm{m})^{-1}$ to $0.04(\Omega \cdot \mathrm{m})^{-1}$. The only exception is a slight extension of the range of $\Delta T$ below zero for $5,10 \mu \mathrm{m}$ diameters and intrafascicular electrodes. Not shown in the figure is that this corresponds to only one case, with all parameters from set $A$. Parameter $\sigma_{o}$ has a clear influence on $A_{\max }\left(D_{i}\right)$ for the intrafascicular electrode only: $A_{\max }\left(D_{i}\right)$ increases when $\sigma_{o}$ increases from the value of set $A$ to the value of set $B$. $T_{i, i+1}$ increases with increasing $\sigma_{o}$ in all cases for the extraneural electrode.

Increasing $\sigma_{\text {iz }}$ from the set $A$ values (low intrafascicular anisotropy) to those in set $B$ (higher anisotropy) results in a lower geometrical spread $S_{i}$, as was the case for decreasing $\sigma_{e}$. However, increased anisotropy $\left(\sigma_{\mathrm{iz}} / \sigma_{\mathrm{ir}}\right)$ increases $A_{\max }(D)$, corresponding to the effect of increasing $\sigma_{e}$.

Increasing $r_{i}$ results in an increased $A_{\max }\left(D_{i}\right)$ and a decreased $S_{i}$ for both electrode configurations, but a decreased $T_{i, i+1}$ for the intrafascicular electrode configuration only.

An increase of $r_{o}-r_{i}$ from 0.05 to $0.5 \mathrm{~mm}$ resulted in increased $A_{\max }\left(D_{i}\right)$ and decreased $T_{i, i+1}$ for the extraneural electrode.

When considering all combinations of model parameters, relations between $S_{i}$ and $T_{i-1, i}$ and between $S_{i}$ and $T_{i, i+1}$ for intrafascicular electrodes can be observed in Fig. 7(a): $T_{i-1, i}$ and $T_{i, i+1}$ decreased with decreasing $S_{i}$. This means that more pronounced inverse recruitment must be expected to coincide with a decreased geometrical spread $S_{i}$ of recruited nerve fibers inside a fascicle in
INTRAFRSCICULAR POINT ELECTRODE

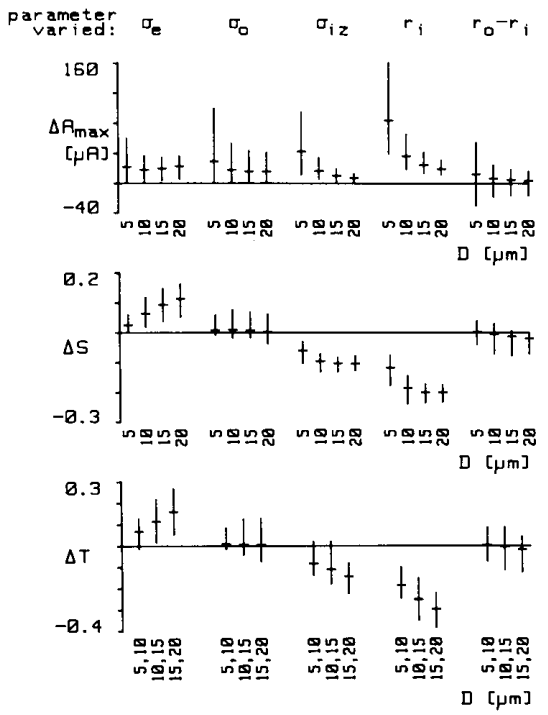

(a)

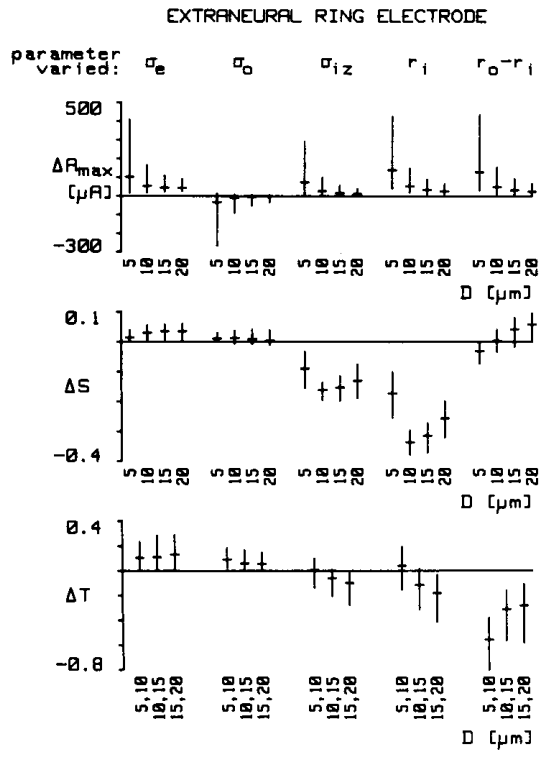

(b)

Fig. 7. The influence of variation of each of the five model parameters on the evaluation variables $A_{m a x}, S_{i}$, and $T_{i, i+1}$. The value change of each evaluation variable is given when one of the model parameters changes from the value in set $A$ to the larger value in set $B$. Both parameter sets are defined in Table I. The vertical bars were drawn between minimum and maximum value change. The horizontal marks give the mean change for all combinations of the other model parameters. (a) Intrafascicular electrode, (b) extraneural electrode.

the case of intrafascicular electrodes. The model did not predict such a relation for extraneural electrodes.

\section{Discussion}

In our model, the simultaneous excitation of many nerve fibers during a stimulation pulse was assumed not to in- 
fluence the conducting properties of the macroscopic volume conductor. To check this, Clark and Plonsey [21] gave a theoretical analysis for unmyelinated nerve fibers. They considered an inactive fiber in a fascicle with many synchronously active fibers, which were assumed to produce an extracellular potential distribution with an amplitude of $10 \mathrm{mV}$. For typical values of membrane resistance and capacitance, only a small transmembrane potential of about $0.3 \mathrm{mV}$ was calculated. We assumed for myelinated fibers this value to be not significantly higher.

A further assumption essential in both the McNeal model and our version is that thicker fibers have a greater internodal length. For small electrode-fiber distances, it can either result in a lower or a higher threshold of excitation, depending on fiber diameter and $L_{0}$. Several investigators experimentally found a larger internodal length for thick nerve fibers than for thin nerve fibers [22]. However, whether the relation between nerve-fiber diameter and internodal length is strictly linear is not certain [22].

Veltink et al. [6], [7] observed a mixed order of recruitment for intrafascicular stimulation by measuring distances between peak-twitch forces achieved for increasing stimulation amplitudes. These distances, corresponding to single-motor unit contributions, did not strictly decrease with every additionally recruited unit, pointing to a nonstrict inverse order of recruitment of the corresponding motoneurons as compared to the physiological order. The model presented describes recruitment in terms of probabilities. At a given stimulation pulse amplitude, e.g., at the indicated value of $9 \mu \mathrm{A}$ for the example of intrafascicular stimulation in Fig. 4(a), the values of $F_{A \mid D}$ give the expected portion of the nerve fibers of given diameter that are recruited. This portion is greater than zero for all four nerve-fiber diameters considered. A nonstrict inverse recruitment is therefore likely to result in realizations of the stochastic process, like during the corresponding intrafascicular stimulation experiments of Veltink et al. [6], [7].

Petrofsky [3] and Fang and Mortimer [4] investigated inverse recruitment by measuring twitch widths, using the property that slow motor units are relatively small compared to faster units and correspond to small diameter motoneuron fibers. For increasing pulse amplitude, a strictly increased muscle twitch width was found for bipolar extraneural stimulation. In terms of the model, this means that the ratio of $F_{A \mid D}$ between nerve fibers of different diameters continuously changes in the direction of smaller nerve fibers for increasing stimulation pulse amplitude. This implies higher $F_{A \mid D}$ for larger diameter nerve fibers. Our simulation results predict such an inverse recruitment order in a probabilistic sense for all cases considered, with minor deviations pointed out in the results. It even applies for intrafascicular stimulation [e.g., Fig. 4(a)] and is not necessarily in contradiction with a mixed order of recruitment as found by Veltink et al. [6], [7]. However, as described in the results, the inverse recruitment is predicted to be more pronounced for extraneural than for intrafascular electrodes in all simulated cases, especially for the larger of both simulated distances $r_{o}-r_{i}$ between electrode and fascicle in the extraneural case.

Solomonow et al. [5] found soleus muscle, predominantly consisting of relatively small-sized motor units, to have a higher stimulation threshold than gastrocnemius muscle, consisting mainly of large units, when stimulating with a bipolar cuff electrode. Soleus thresholds appeared to be lower than gastrocnemius thresholds when restoring physiological order by high-frequency blocking. These findings correspond to our simulation results for extraneural electrodes: $A_{\min }$ increased with increasing diameter for all cases for the extraneural electrode [as shown for one case in Fig. 4(b)].

Blair and Erlanger [2] found a strict inverse order of recruitment for extraneural stimulation of a frog's sciatic nerve near the spinal cord. Nerve potentials were measured as far as possible distal to the point of stimulation. Different nerve fiber diameters could be identified by differences in conduction times of action potentials. Blair and Erlanger determined strength-duration curves for excitation thresholds of nerve fibers with different conduction times. In this way, nerve fibers with a large diameter were always found to have a higher stimulation threshold than nerve fibers with small diameter. For several reasons, these measurements of strict inverse recruitment can still be in correspondence with the results of our model:

1) For extraneural stimulation our model predicts pronounced inverse recruitment, especially for low extraneural conductivity and large distances between electrode and fascicle. For the case of Fig. 4(b) at the stimulation pulse amplitude indicated $(30 \mu \mathrm{A})$, almost all nerve fibers of $15 \mu \mathrm{m}$ diameter most be expected to be recruited, while recruitment of $10 \mu \mathrm{m}$ diameter nerve fibers just starts.

2) When nerve fibers of arbitrary small diameter difference are always observed to be recruited in inverse order, the distribution functions $F_{\boldsymbol{A} \mid \boldsymbol{D}}$ need to be step functions. Blair and Erlanger [2] could only distinguish different diameter nerve fibers when differences in conduction times were larger than the duration of a single action potential. Therefore, the distribution functions $F_{A \mid D}$ [e.g., Fig. 4(b)] need not be step functions in order to be in correspondence to the strict inverse recruitment order found by Blair and Erlanger.

3 ) In the curves giving the relation between conduction velocity and threshold, Blair and Erlanger [2] indicate some spread in threshold over nerve fibers having the same conduction velocity.

4) The experimental data presented by Blair and Erlanger [2] indicate a much larger change of excitation threshold with a change of conduction velocity for small than for large conduction velocities. This corresponds to smaller distances between $F_{A \mid D}$ curves for thicker nerve fibers (e.g., between 15 and $20 \mu \mathrm{m}$ diameter) than for thin nerve fibers (e.g., between 5 and $10 \mu \mathrm{m}$ ) in the example of Fig. 4(b), and to a lower differential diameter dependency of $A_{\max }$ for higher nerve-fiber diameters in Fig. 6(b), considering that the relation between nerve-fiber diameter and conduction velocity is almost linear [18]. 


\section{REFERENCES}

[1] E. Henneman, "Recruitment of motoneurons: The size principle," in Motor Unit Types, Recruitment, and Plasticity in Health and Dis ease-Prog. Clin. Neurophysiology, vol. 9, J. E. Desmedt, Ed. Basil, Karger, 1981, pp. 26-60.

[2] E. A. Blair and J. Erlanger, "A comparison of the characteristics of axons through their individual electrical responses," Amer. J. Physiol., vol. 106, pp. 524-564, 1933.

[3] J. S. Petrofsky, "Control of the recruitment and firing frequencies of motor units in electrically stimulated muscles in the cat," Med. Biol. Eng., vol. 16, pp. 302-308, 1978.

[4] Z.-P. Fang and J. T. Mortimer, "A method for attaining natural recruitment order in artificially activated muscles," in Proc. 9th Anu. Conf. IEEE Eng. Med. Biol. Soc., Boston, MA, 1987, vol. 2, pp. $657-658$.

[5] M. Solomonow, R. Baratta, and B. H. Zhou, "Comparative electromyography and biomechanics of orderly and reversed recruitment," in Proc. 9th Annu. Conf. IEEE Eng. Med. Biol. Soc., Boston, MA, 1987, vol. 2, pp. 647-648.

[6] P. H. Veltink, H. J. Hermens, and J. A. van Alsté, "Multielectrode intrafascicular and extraneural stimulation," in Proc. 8th Annu. Conf. IEEE Eng. Med. Biol. Soc., Dallas, TX, 1986, pp. 690-693.

[7] P. H. Veltink, J. A. van Alsté, and H. B. K. Boom, "Multielectrode intrafascicular and extraneural stimulation," Med. Biol. Eng. Com put., to be published.

[8] J. S. Petrofsky and C. A. Phillips, "Impact of recruitment order on electrode design for neural prosthetics of skeletal muscle," Amer. $J$. Phys. Med., vol. 60, pp. 243-253, 1981.

[9] M. Solomonow, "External control of the neuromuscular system," IEEE Trans. Biomed. Eng., vol. BME-31, pp. 752-763, 1984.

[10] J. Holle, M. Frey, H. Gruber, H. Kern, H. Stöhr, and H. Thoma, "Functional electrostimulation of paraplegics. Experimental investigations and first clinical experience with an implantable stimulation device,"'Orthopedics, vol. 7, pp. 1145-1155, 1984.

[11] J. S. Petrofsky, "Sequential motor unit stimulation through periph eral motor nerves in the cat," Med. Biol. Eng. Comput., vol. 17, pp. 87-93, 1979.

[12] D. R. McNeal and B. R. Bowman, "Selective activation of muscles using peripheral nerve electrodes," Med. Biol. Eng. Comput., vol. 23, pp. 249-253, 1985.

[13] P. Talonen, G. Baer, M. Huhti, and V. Häkkinen, "Control of muscle force by sequential motor unit stimulation of peripheral nerves,' in Proc. XIV Int. Conf. Med. Biol. Eng. and VII Int. Conf. Med. Physics, 1985, pp. 396-397.

[14] P. H. Veltink, J. A. van Alsté, and H. B. K. Boom, "Simulation of intrafascicular and extraneural nerve stimulation," IEEE Trans. Biomed. Eng., vol. 35, pp. 69-75, 1988.

[15] P. H. Gorman and J. T. Mortimer, "The effect of stimulus parameters on the recruitment characteristics of direct nerve stimulation," IEEE Trans. Biomed. Eng., vol. BME-30, pp. 407-414, 1983.

[16] D. R. McNeal, "Analysis of a model for excitation of myelinated nerve," IEEE Trans. Biomed. Eng., vol. BME-23, pp. 329-337, 1976.

[17] B. Frankenhaeuser and A. F. Huxley, "The action potential in the myelinated nerve fiber of xenopus laevis as computed on the basis of voltage clamp data," J. Physiol., vol. 171, pp. 302-315, 1964.

[18] D. F. Stegeman and J. P. C. van de Weerd, "Modeling compound action potentials of peripheral nerves in situ: I. Model description Evidence for a nonlinear relation between fiber diameter and velocity," Electroencephalogr. clin. Neurophysiol., vol. 54, pp. 436-448, 1982
[19] L. A. Geddes and L. E. Baker, "The specific resistance of biological material-A compendium of data for the biomedical engineer and physiologist," Med. Biol. Eng., vol. 5, pp. 271-291, 1967.

[20] S. Sunderland, Nerves and Nerve Injuries. Edinburgh and London: Livingstone, 1968

[21] J. W. Clark and R. Plonsey, "Fiber interaction in a nerve trunk," Biophys. J., vol. 11, pp. 281-294, 1971.

[22] P. K. Thomas and J. Ochoa, "Microscopic anatomy of peripheral nerve fibers," in Peripheral Neuropathy, vol. l, P. J. Dyck, P. K. Thomas, E. H. Lambert, and R. Bunge, Eds. Philadelphia, PA: Saunders, 1984, pp. 39-96.

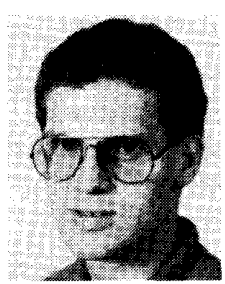

Peter H. Veltink was born in Groenlo, The Neth erlands, in 1960. He studied electrical engineering at the University of Twente, The Netherlands, and received the M.S. degree in 1984 and the Ph.D. degree in 1988 .

Currently, he is a Member of the Biomedical Engineering Division in the Department of Electrical Engineering, University of Twente, and is engaged in the research of neuromuscular stimulation for rehabilitation of spinal cord injured patients, which is carried out in cooperation with the Roessingh Rehabilitation Center, Enschede. His research interests are nerve stimulation and control systems for functional neuromuscular stimulation.

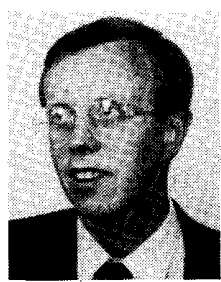

Jan A. van Alsté (S'75-S'83-M'83-M'84) was born in Hengelo, The Netherlands, in 1946. After polytechnics and three years of industrial electronic development, he joined the Biomedical Engineering Division of the Twente University of Technology where he received the M.S. degree in electrical engineering in 1978 and the Ph.D. degree in 1984.

His research interests include exercise ECG analysis, heart assist devices, and functional neuromuscular stimulation. He is part of the rehabilitation engineering cooperation between the University of Twente and the Roessingh Rehabilitation Center, Enschede, The Netherlands. Currently, he is Director of the Coordination Center for Biomedical Engineering, University of Twente, The Netherlands.

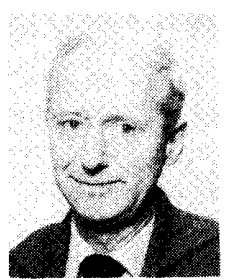

Herman B. K. Boom was trained as a Medical Physicist at the University of Utrecht, The Netherlands, where he received the Ph.D. degree in 1971

He joined the Departments of Medical Physics and Medical Physiology where he was engaged in research in the field of cardiac mechanics and taught physiology and biophysics. Since 1976 he has occupied the Chair of Medical Electronics in the Department of Electrical Engineering, University of Twente, The Netherlands. His research interests are cardiovascular system dynamics, bioelectricity, and rehabilitation technology. 